\title{
Phased Array Measurements from Landing Gear Models
}

\author{
Alexander R. Quayle ${ }^{1}$ Ann P. Dowling ${ }^{2}$, Holger Babinsky ${ }^{3}$, William R. Graham ${ }^{4}$, Yu Liu ${ }^{5}$ \\ University of Cambridge, England
}

\begin{abstract}
Landing gear is now well known to be a major source of aircraft noise. Several studies have also identified the importance of surface details in the noise generated by landing gears at high frequencies. However, the basic mechanisms of noise generation at lower frequencies are less well understood. In this study, we examine the effect of changes to the overall gear layout on the noise produced. $1 / 12^{\text {th }}$ scale models containing only the wheels, axles and main struts have been studied using two nested, 48-microphone arrays in the closed-section Markham wind tunnel at Cambridge University. Local fairings have also been added to isolate and identify individual noise sources. Results indicate that shape and placement of the wheels can affect the overall noise level by at least $6 \mathrm{~dB}$ on simplified four wheel models. Changes in geometry were also found to substantially affect high frequency sources close to the main oleo, suggesting that noise sources from conventional, fully dressed gears might be equally susceptible to small changes in the overall design.
\end{abstract}

\section{Nomenclature}

$\begin{array}{ll}S P L & =\text { Sound pressure level, } \mathrm{dB}\left(\mathrm{ref} 2 \times 10^{-5} \mathrm{~Pa}\right) \\ d & =\text { wheel diameter, } \mathrm{m} \\ M & =\text { Mach no } \\ r & =\text { Source }- \text { observer radius } \\ f & =\text { frequency, } \mathrm{Hz} \\ V & =\text { flow speed, } \mathrm{m} / \mathrm{s}\end{array}$

\section{Introduction}

T ANDING gear continues to be one of the main sources of airframe noise due to its large size, complex Leometry, bluff nature and position on the aircraft. Considerable work has been done to understand the noise sources associated with the gear ${ }^{1-6}$ and both empirical and semi-empirical models have been developed to predict the noise generated by landing gears ${ }^{7,8}$.

Proposed methods of reducing landing gear noise have typically centered around the fine details which are attached to the gear surface ${ }^{2,4}$. These produce relatively high frequency sound $(1-3 \mathrm{kHz})$, which is particularly irritating to the human ear. Local fairings on conventional landing gears have achieved noise reductions of around 6dBA by eliminating some of this high frequency component ${ }^{2}$. In addition to the high frequency details, the torque link has also been identified as a major noise source ${ }^{5}$. However, even with removal of these dominant noise sources, the landing gear is still predicted to be a major noise source on low noise aircraft ${ }^{1}$.

The aim of this work is to understand the effect of the basic landing gear geometry on noise generation mechanisms. Published work suggests there is a link between the spacing of wheels on a four wheel landing gear and the location of the main noise sources ${ }^{6}$. Significantly changing the spacing between the wheels was found to affect which sources dominated the overall bogie noise but did not offer a clear route to eliminating noise from the models ${ }^{6}$. Other studies have highlighted the importance of wheel - wheel interactions between adjacent front and rear wheels as a mechanism of noise generation ${ }^{5,9}$.

\footnotetext{
${ }^{1}$ Research Student, Cambridge University Engineering Department, Cambridge, England. Member AIAA

${ }^{2}$ Professor, Cambridge University Engineering Department, Cambridge, England. Member AIAA

${ }^{3}$ Reader in Aerodynamics, Cambridge University Engineering Department, Cambridge, England. Senior Member AIAA

${ }^{4}$ Senior Lecturer, Cambridge University Engineering Department, Cambridge, England. Member AIAA

${ }^{5}$ Research Student, Cambridge University Engineering Department, Cambridge, England. Member AIAA
} 
In previous experiments ${ }^{1}$, the edge radius applied to model scale wheels was found to be important at frequencies of $2-4 \mathrm{kHz}(200-400 \mathrm{~Hz}$ full scale). This source was found to be close to the front of the rear wheels, suggesting an impingement of the flow from the front wheel on the adjacent rear wheel. This interaction was seen to be a limiting factor in the effectiveness of extensive fairings, so that sources at these frequencies might dominate the spectrum of a low noise landing gear ${ }^{1}$.

In the present study we examine four- and twin- wheel configurations using models with adjustable wheel position and shape. In addition, sources associated with downstream components are isolated by filling in the gaps between adjacent wheels and adding fairings between the axles. Through identifying the interactions between components on the simplified model, it is hoped that an understanding of more complex geometries can be achieved.

\section{Experimental method}

\section{A. Experimental measurements}

Phased array measurements were made in the Markham closed section wind tunnel at the University of Cambridge. The tunnel has a working section of $1.6 \mathrm{~m} \mathrm{x} 1.2 \mathrm{~m}$ and has been fitted with a pair of nested microphone arrays, each with 48 microphones which were used to capture the noise data ${ }^{10}$. Experiments were conducted at $50 \mathrm{~m} / \mathrm{s}$, providing Reynolds numbers of $4.0 \times 10^{5}$ based on wheel diameter. Models were mounted in the centre of the tunnel, with the oleo / beam junction $0.6 \mathrm{~m}$ above the nested acoustic arrays (Figure 1). The high frequency array was sampled continuously for $180 \mathrm{~s}$ at $120 \mathrm{kHz}$ and the low frequency array for $600 \mathrm{~s}$ at $30 \mathrm{kHz}$.

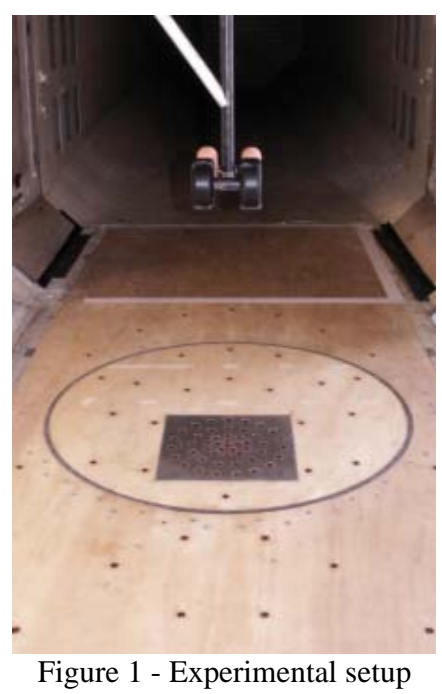

The acoustic array and beamforming methods are similar to those described previously ${ }^{1}$. Autopowers were removed from the cross power matrix before beamforming ${ }^{11}$. The CLEAN algorithm was employed to quantify the noise sources associated with the model, whilst eliminating the effect of background noise ${ }^{1,12,13}$. Results were repeatable to approximately $1 \mathrm{~dB}$ per third octave band.

A light density, $0.25 \mathrm{~mm}$ ballotini transition trip was applied over the surface of the front axle and main strut to $65^{\circ}$ from the stagnation point, in order to represent full scale flow. This method of tripping was verified using a static pressure tapping at the rear of a similar cylinder in a separate experiment. A similar, medium density, trip was applied over a smaller region (in anticipation of the higher Reynolds number) on the front wheels at around $60^{\circ}$ from the stagnation point. 


\section{B. Configurations examined}

\section{Four wheel landing gears}

Figure 2 shows the model setup for the four wheel landing gear. All wheels have a diameter of $0.1 \mathrm{~m}$ (approximately $1 / 12^{\text {th }}$ scale). Spacers were used to increase the lateral separation between the wheels and two different axle beams were used to increase the streamwise spacing. These adjustments allowed the basic gear geometry to cover the range of shapes typically observed on large commercial aircraft. Models were constructed with the deliberate aim of removing surface discontinuities and fine details. Joints were designed to be internal to the gear and securing bolts were embedded and smoothly covered. A side strut was also added at a height of 1.5 wheel diameters above the oleo / beam junction to provide stability whilst avoiding any undesirable interference with the remaining model.

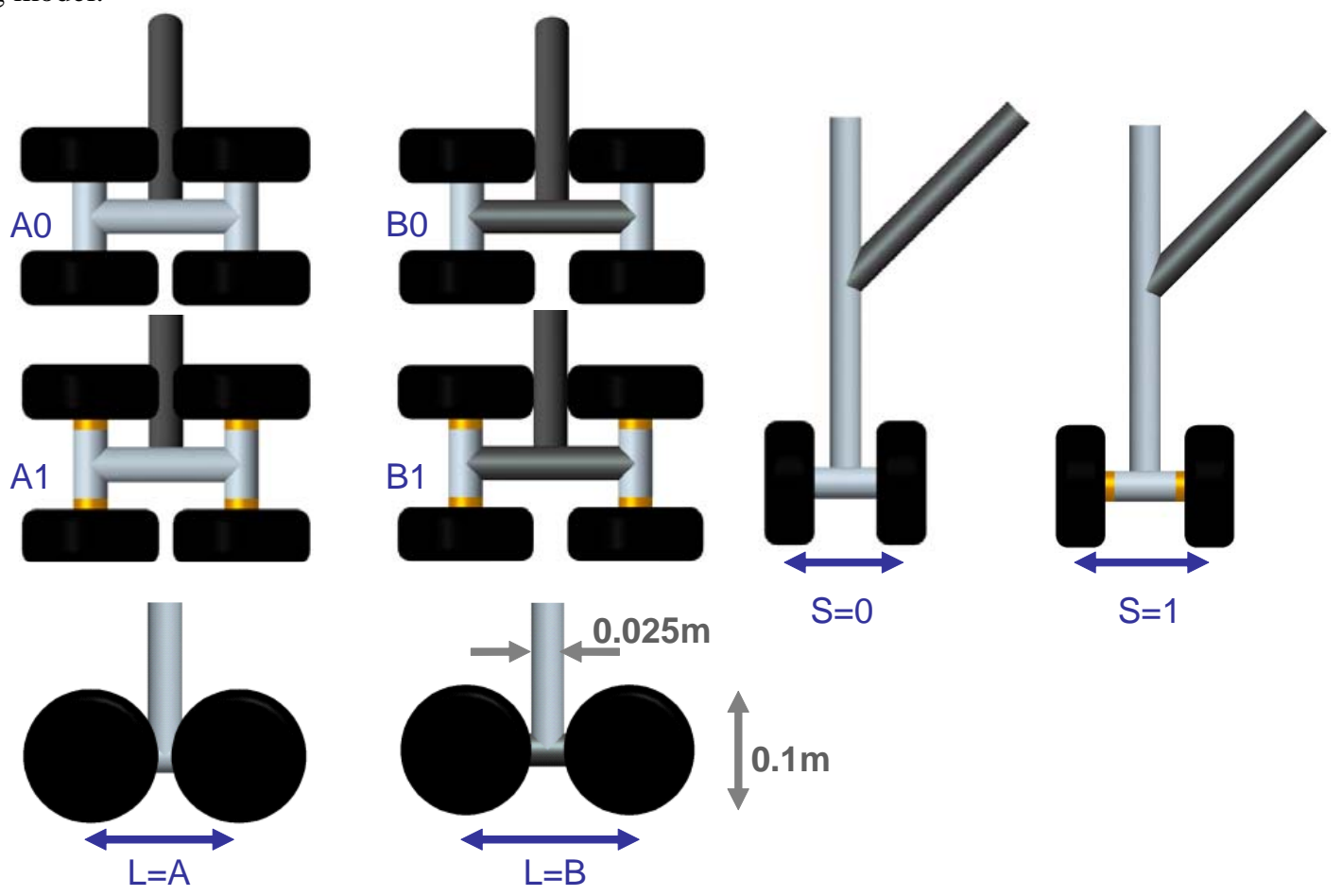

Figure 2. Layout of the four wheel models (spacing dimensions are given in table 1)

Configuration

A0

A1

B0

B1

Co (2whl)

C1 (2whl)

\section{Longitudinal Spacing (L), wheel diameters}

1.1

1.1

1.25

1.25

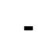

$-$

$-$
Spanwise / Lateral Spacing (S), wheel widths

2.75

2.25

2.75

2.25

2.75

Table 1. Wheel spacing of the experimental models

\section{Isolation of the wheel - wheel interaction and axle noise sources}

In order to understand the importance of the gap between the wheels as a source of noise, a 'track' configuration was examined, which retained the basic landing gear shape but with a continuous track instead of adjacent wheels (Models T0/T1, Figure 3). To further investigate noise sources associated with the axles, a fairing was installed 
between the axles of model T0 to form model T0F1. Together, these configurations give an estimate of the lowest noise achieveable from a four wheel landing gear, and provide some insight to the noise generated by interaction between components of the gear.

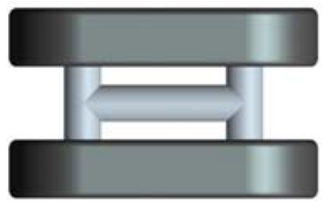

TO

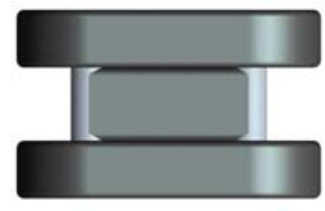

TOF1

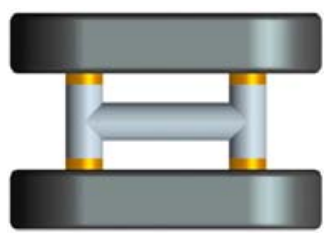

T1

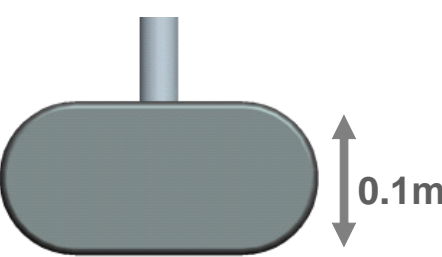

Side view

Figure 3 - Diagram of the 'Track' gear

\section{Wheel edge geometry}

In addition to evaluating different spacing between the landing gear wheels, the configurations of Figure 2 were also examined with different edge radii applied to the model wheels. Three different edge radii were applied to the model wheels, as shown in Figure 4. The $10 \mathrm{~mm}$ radius is approximately representative of wheels at full scale.

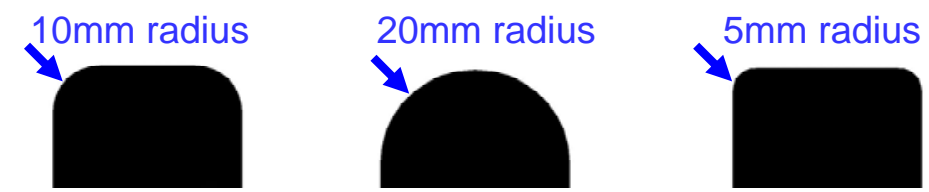

Figure 4 - Edge radii applied to the model landing gear wheels

\section{Twin wheel landing gears}

In the final configuration, the effect of wheel spacing on a twin wheel gear was also examined (Figure 5). Details of the geometry can also be found in Table 1 .

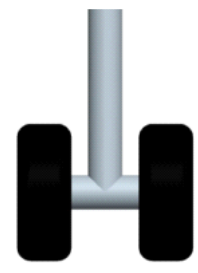

2-CO

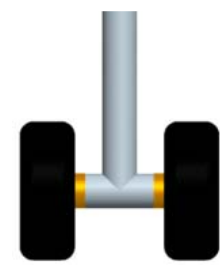

2-C1

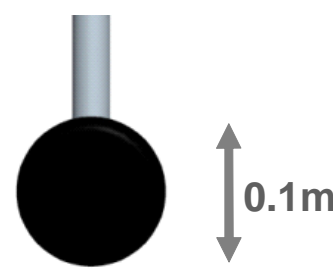

Side view

Figure 5 - Twin wheel landing gear models

\section{Results}

\section{A. Landing gear layout and wheel edge radius}

Noise data presented in the following figures are for overhead noise at model scale and in 1/3 octave bands. Measurements below $6 \mathrm{kHz}$ model scale are taken from measurements with the larger, low frequency array whereas those above $6 \mathrm{kHz}$ are from the smaller, high frequency array (Figure 1).

Figure 6 shows the effect of a change in model geometry for different layout configurations with a 10mm edge radius on the model wheels. Configuration A0 is used as a reference level, with noise measurements for models A1, B0, B1 shown as differences in 1/3 octave SPL resulting from the change in model geometry. Sample source maps are also given in Figure 7 for configuration A0. 
Figure 8-Figure 11 demonstrate the effects of $10 \mathrm{~mm}$ and $20 \mathrm{~mm}$ edge radius wheels in different combinations. In each case, the values shown are changes in landing gear noise from a baseline configuration - here taken to be Model A0 with $10 \mathrm{~mm}$ edge rounding on all the wheels. The source levels can therefore be compared directly between figures.

Figure 12-Figure 16 examine configurations with the $5 \mathrm{~mm}$ edge radius (Figure 4). Values are presented on a common scale to those from previous figures. Corresponding source maps are also shown in Figure 13, Figure 15.
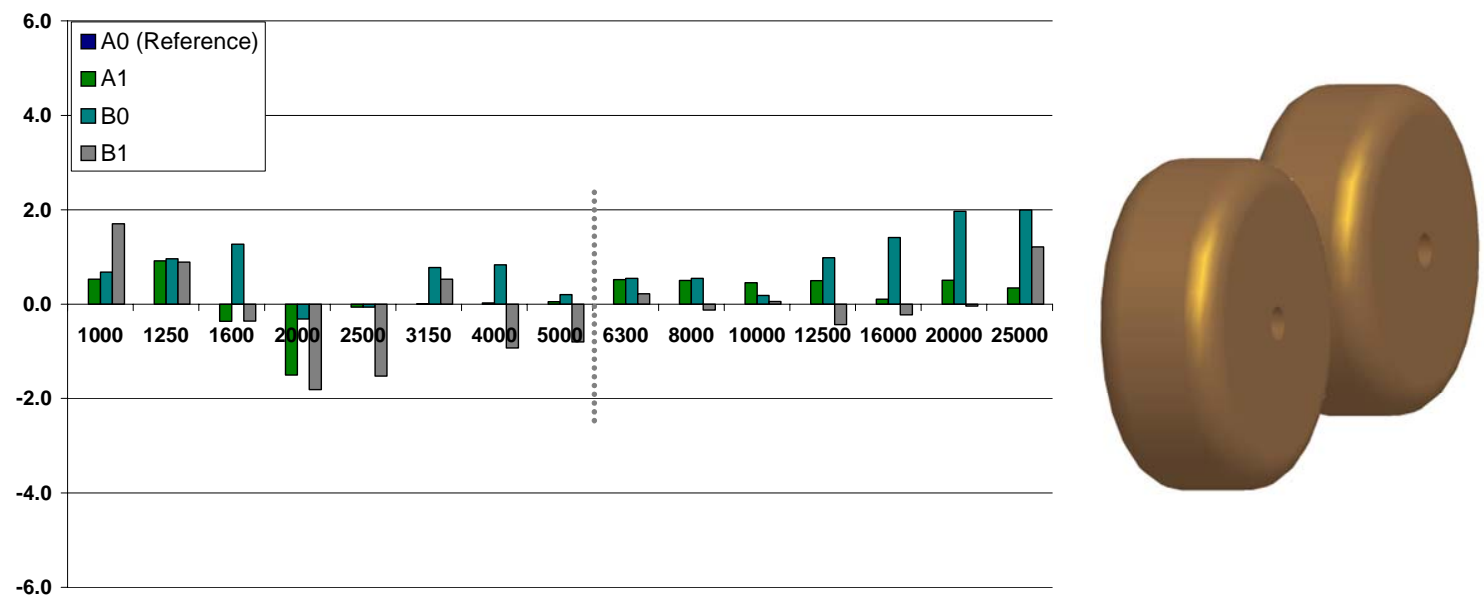

Figure 6 - Overhead noise levels from models A0, A1, B0, B1 with 10mm a wheel edge radius
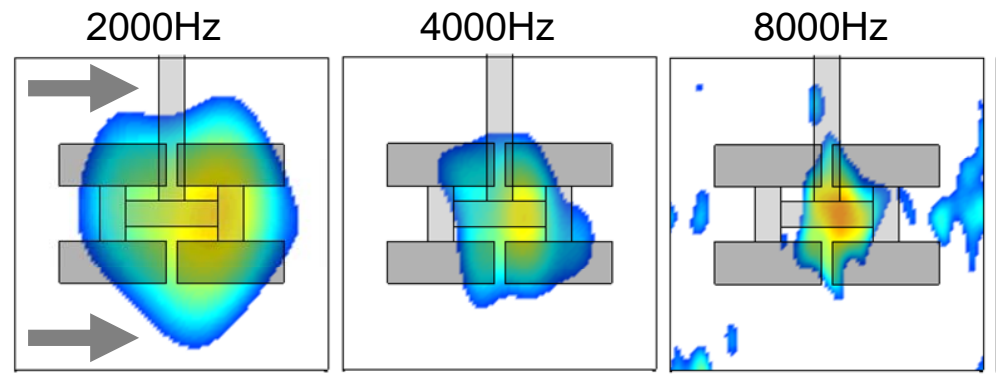

$12,500 \mathrm{~Hz}$

Figure 7 - Source maps showing the noise source location on Model A0 (flow from left to right)
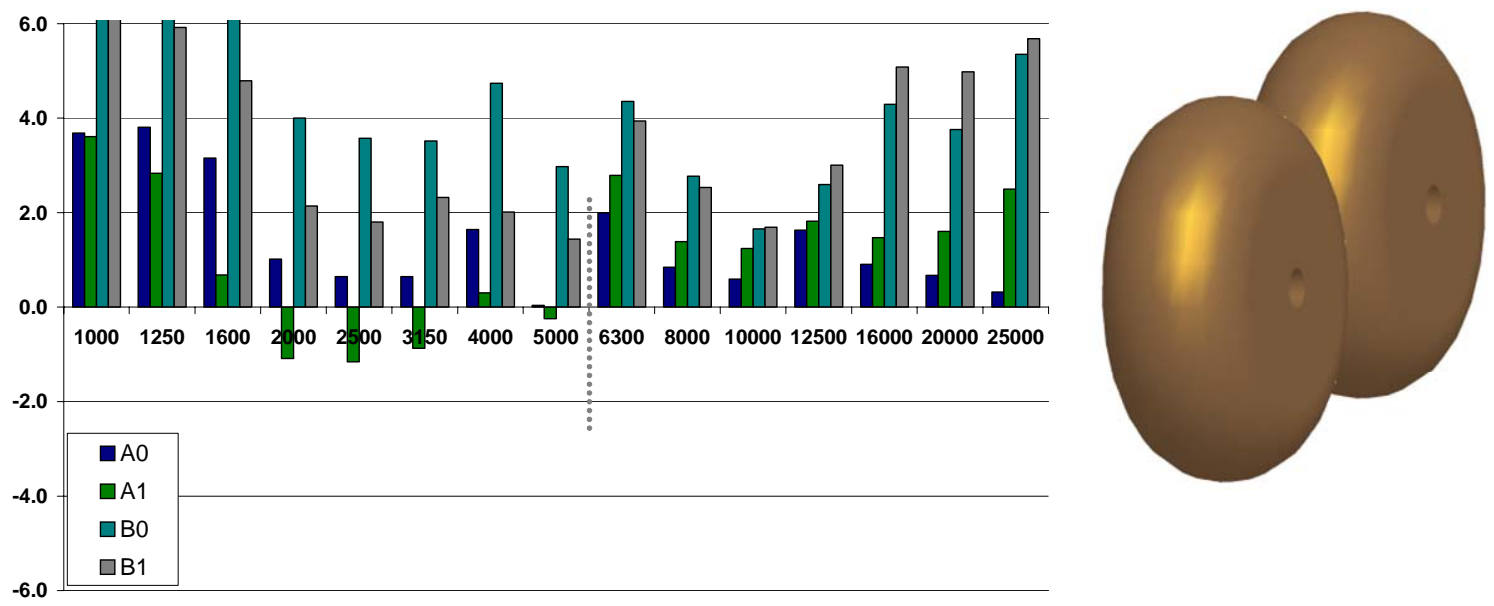

Figure 8 - Models A0, A1, B0, B1 with 20mm a wheel edge radius 

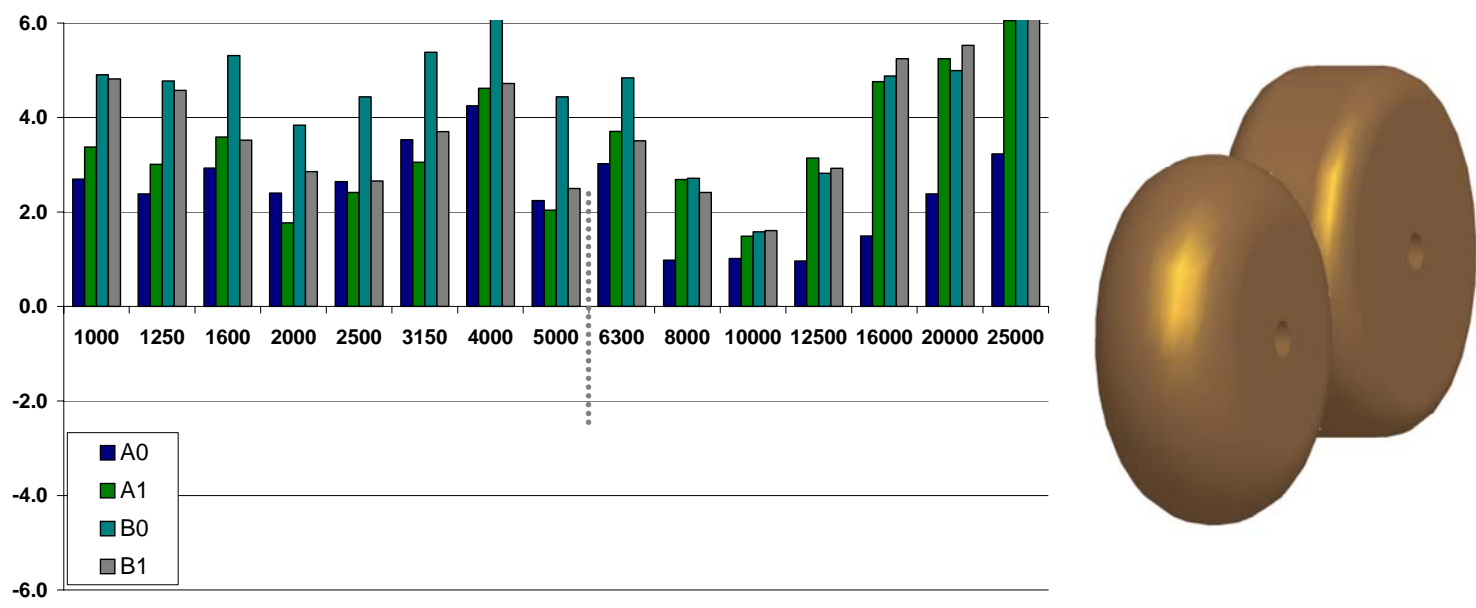

Figure 9 - Models A0, A1, B0, B1 with 20mm (front) and 10mm (rear) edge radius
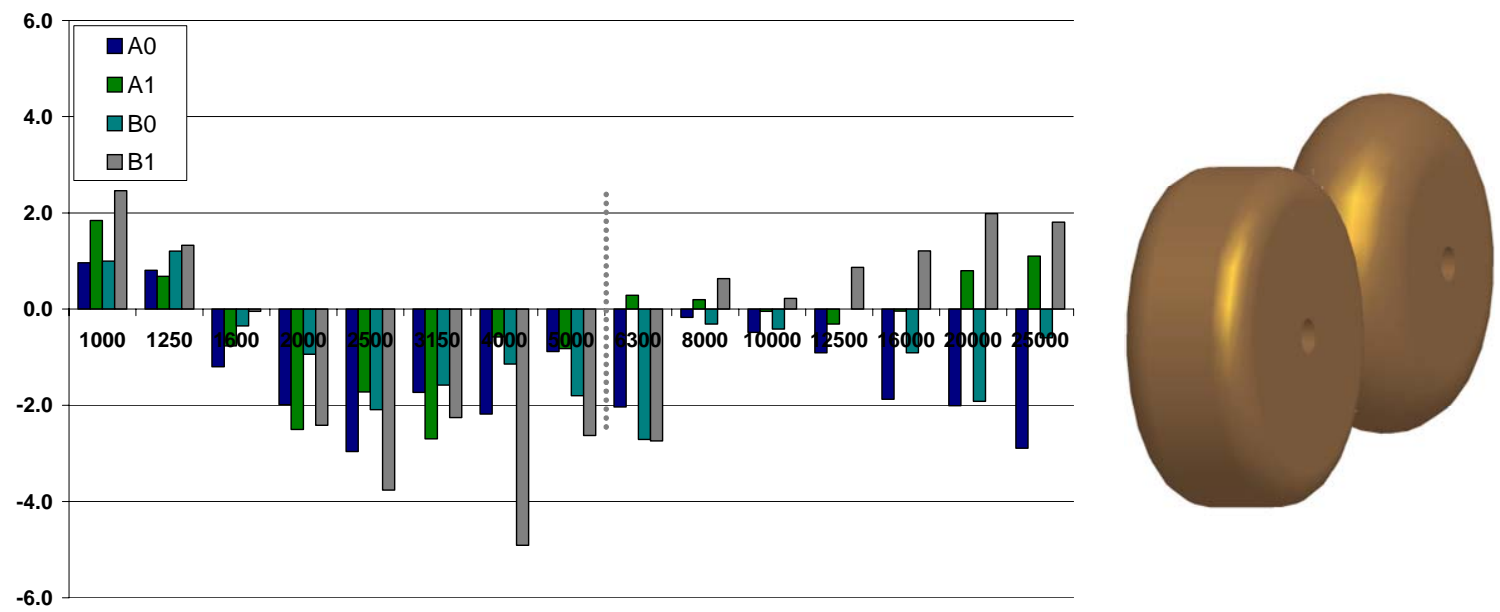

Figure 10 - Models A0, A1, B0, B1 with 10mm (front) and 20mm (rear) edge radius
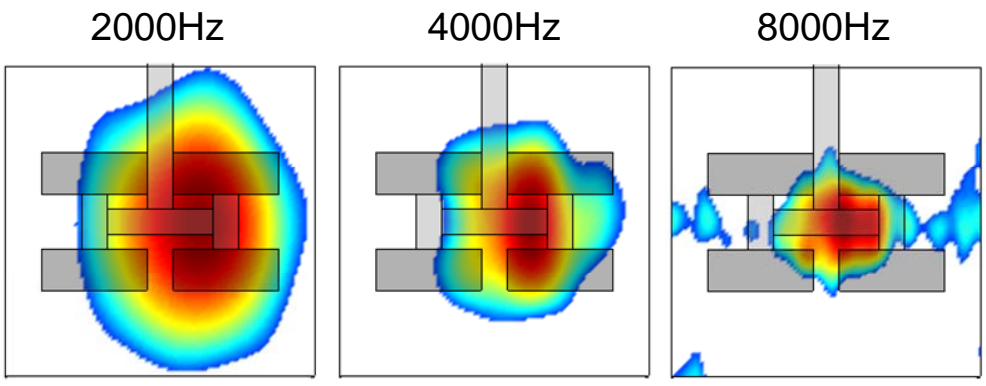

$12,500 \mathrm{~Hz}$

Figure 11 - Source maps for model B0, Edge radius Front 20mm, Rear 10mm (Figure 9) 

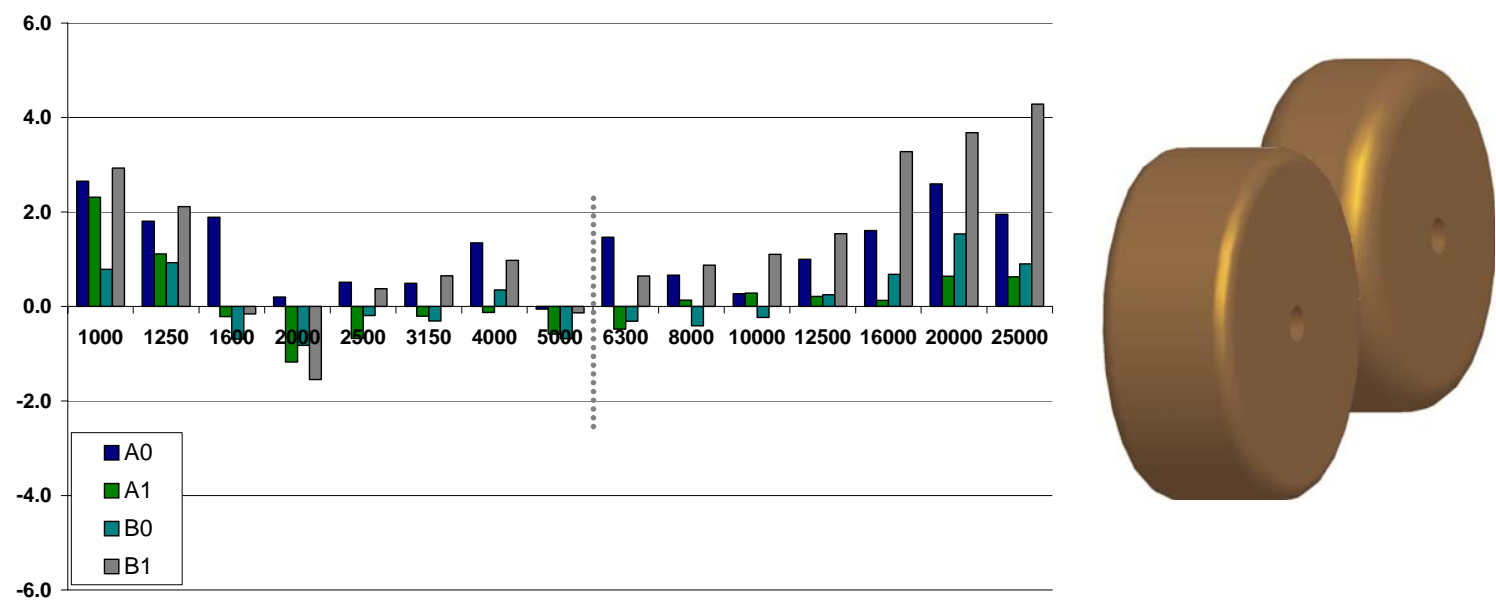

Figure 12 - Models A0, A1, B0, B1 with 5mm (front) and 10mm (rear) edge radius
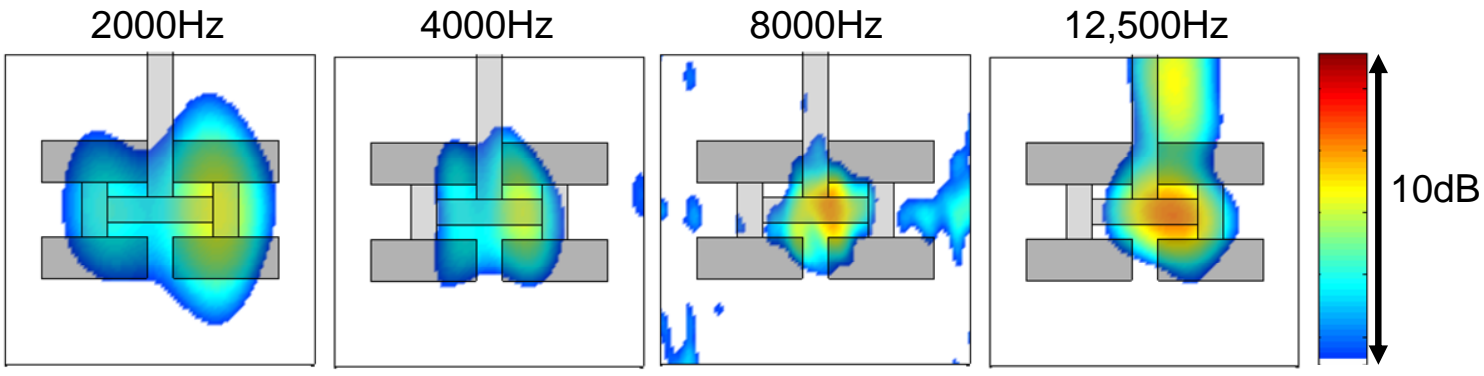

Figure 13 - Source maps for Model B0 with 5mm (front) and 10mm (rear) edge rounding (Figure 12)
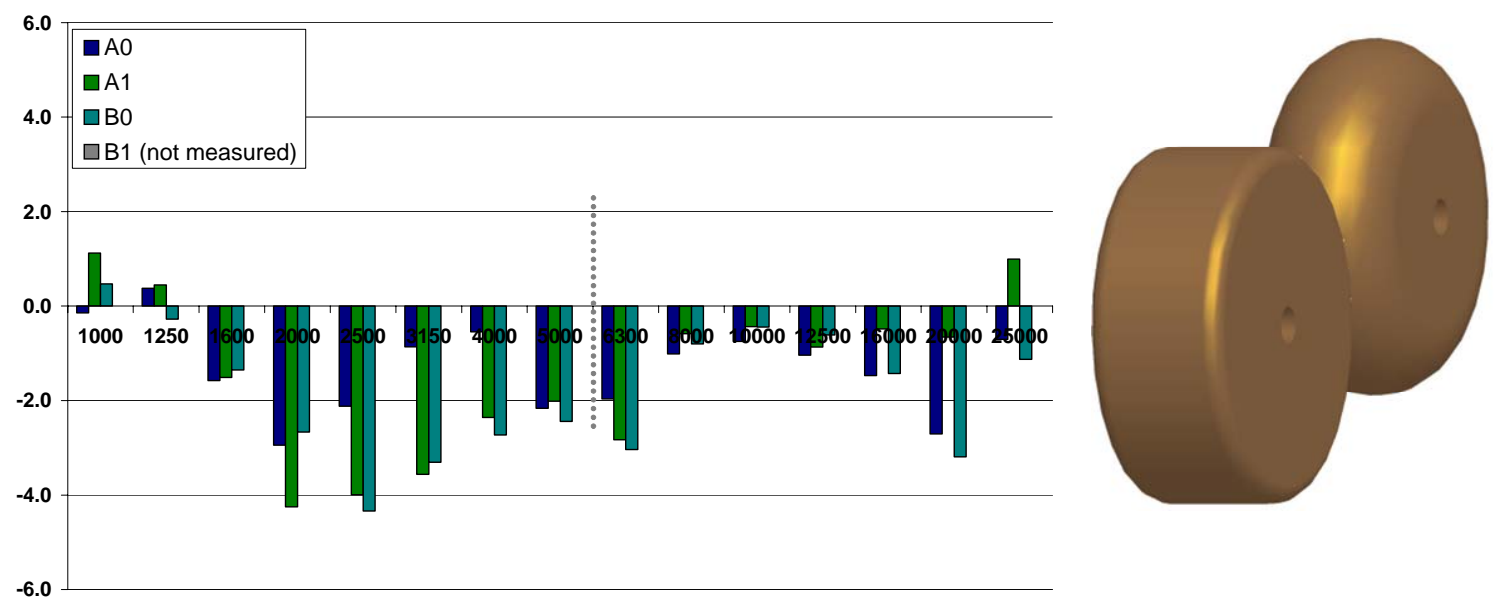

Figure 14 - Models A0, A1, B0 with 5mm (front) and 20mm (rear) edge radius 

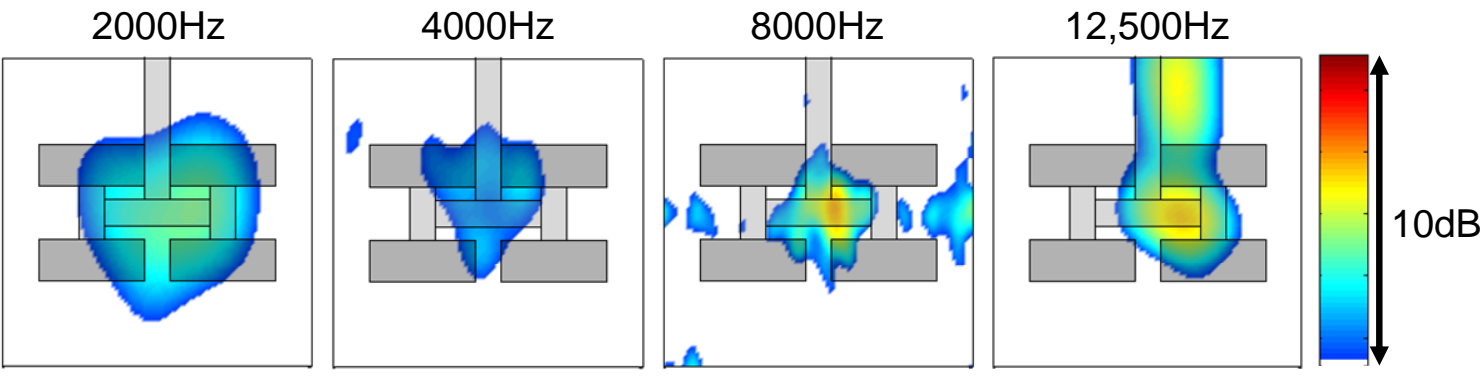

Figure 15 - Source maps for Model B0 with 5mm (front) and 20mm (rear) edge rounding (Figure 14)
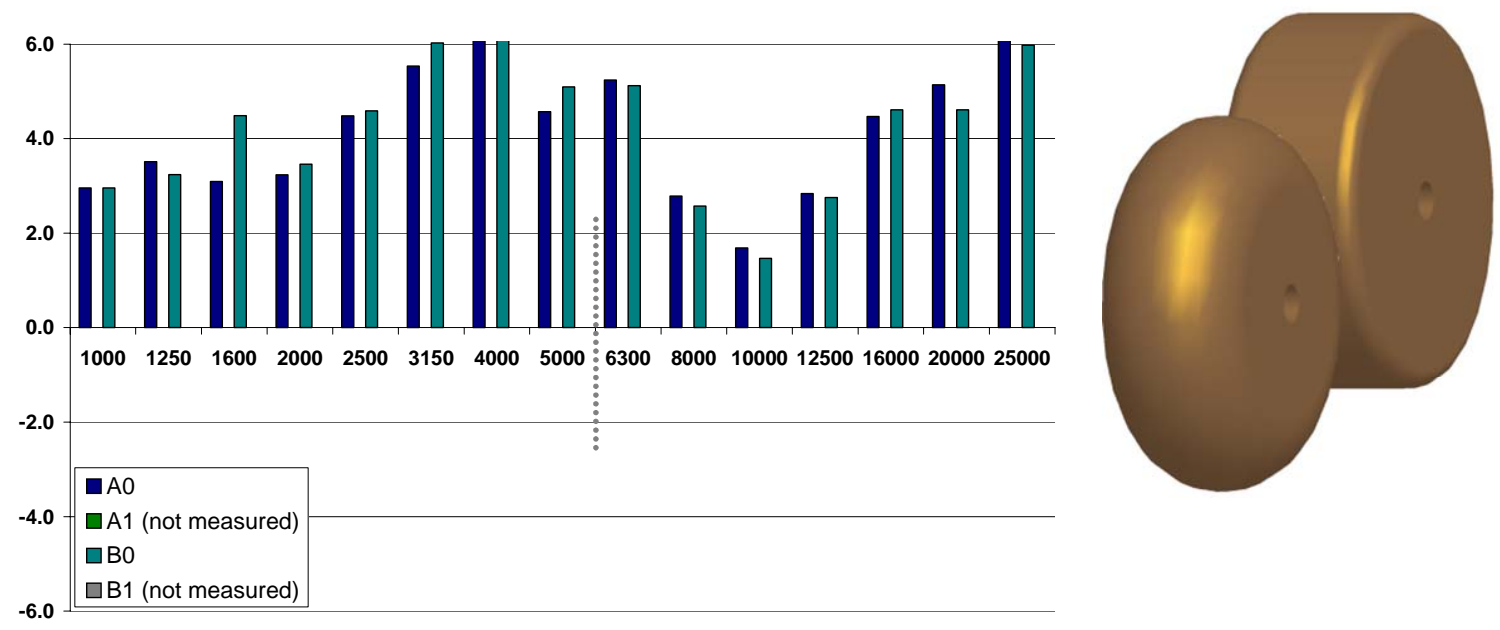

Figure 16 - Models A0, B0 with 20mm (front) and 5mm (rear) edge radius

\section{Isolation of individual sources}

Figure 17 shows the effect of using tracks instead of a pair of adjacent wheels, in order to isolate the effects of the gap between wheels. Tracks were fitted in the same configuration as models A 0 and A1 of Figure 2. Models fitted with tracks did not include a side strut. Figure 18 captures the effect of the side strut in isolation for models A0, A1, B0, B1. In addition, an axle fairing was fitted to model T0 to create configuration T0F1 (Figure 3). This fairing eliminates sources associated with axle interaction and also blocks noise sources from the oleo / wheel interaction from propagating to an observer below. As for previous data, the values in Figure 17 are shown as differences between the A0 configuration of Figure 6 and the data measured in this experiment.
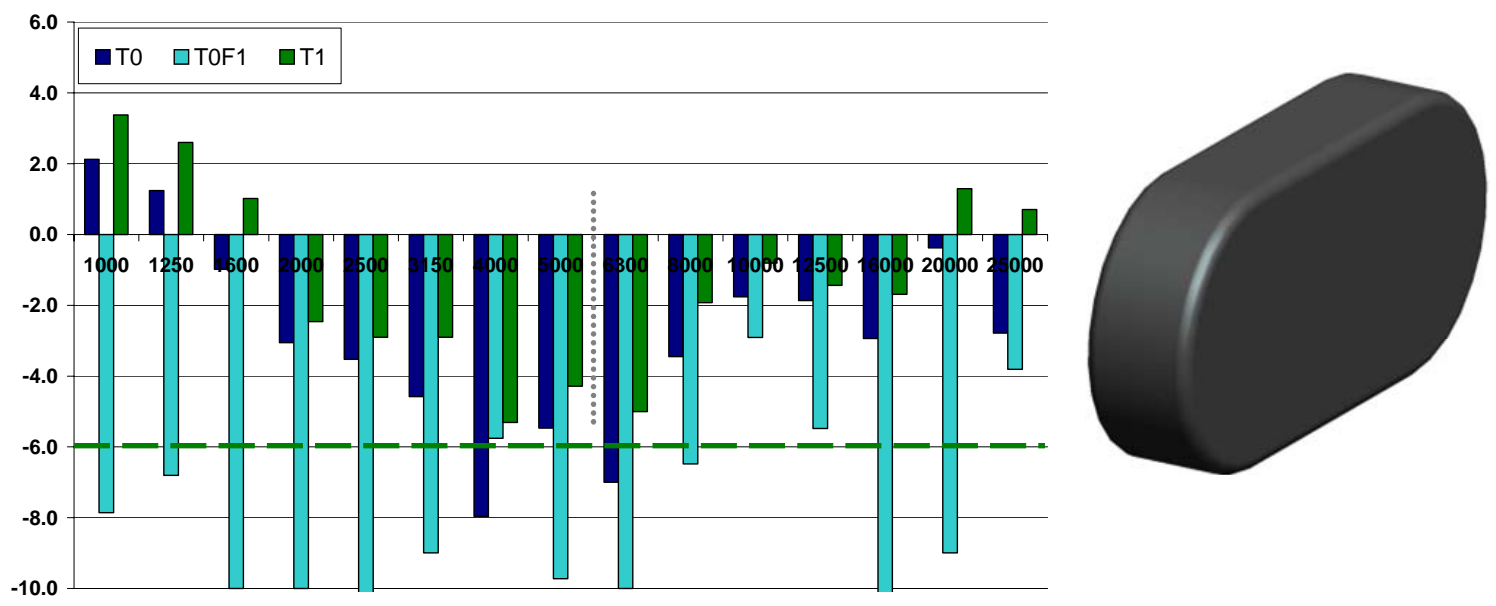

Figure 17 -- Models T0, T0F1 and T1 (note: increase in scale) 

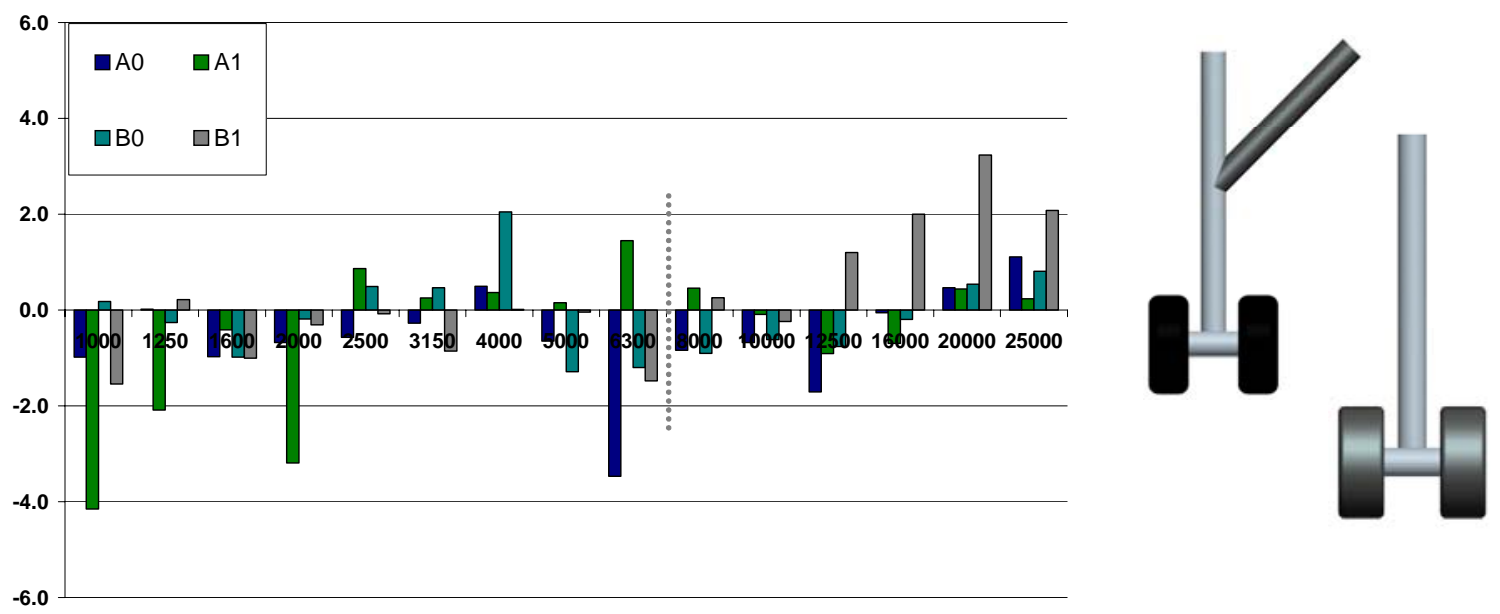

Figure 18 - Effect of the side-strut on Models A0, A1, B0, B1

\section{Twin wheel landing gears}

Finally, results are presented for the twin wheel landing gears (Figure 19). Measurements are again compared with a baseline configuration of the four wheel model in configuration A0 (Figure 2).
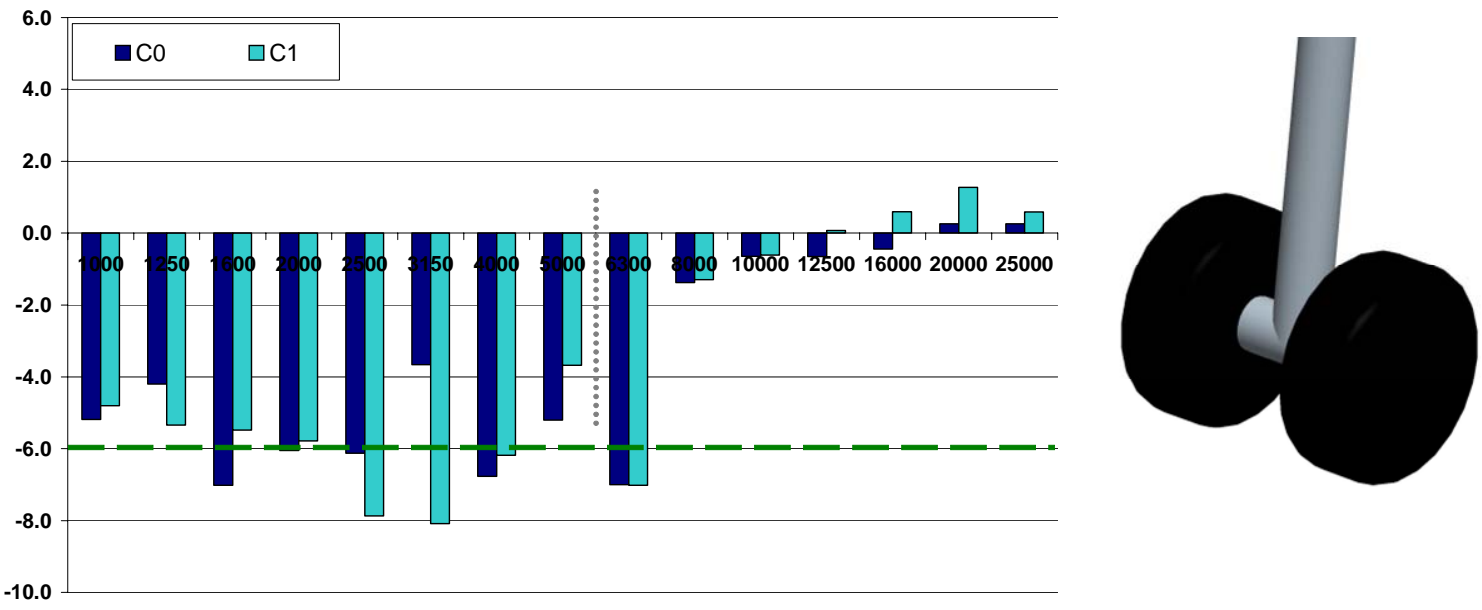

Figure 19 - Twin wheel landing gear noise (note: increase in scale)

\section{Discussion}

\section{A. Analysis of Results}

\section{Wheel edge radius and landing gear layout}

Figure 6 examines the different four wheel geometries of Table 1 with a $10 \mathrm{~mm}$ wheel edge radius. The experimental error in each third octave band is approximately $1 \mathrm{~dB}$, so that the results suggest only a small change in overall landing gear noise between the four geometries examined. Figure 7 shows the approximate source locations at different frequencies for configuration A0. Source locations on other configurations were found to be similar.

When the edge radius is increased to $20 \mathrm{~mm}$ (Figure 8 ), there is a $2-4 \mathrm{~dB}$ difference between measurements with the short and long axle beams (configurations A / B), suggesting that increased streamwise separation of the wheels has a marked effect on the overall noise level. Increasing the spanwise separation of the wheels (configuration 1) 
also tends to decrease the overall noise level in the $2-4 \mathrm{kHz}$ range by approximately $2 \mathrm{~dB}$. As a result, the loudest configuration is model B0 and the lowest noise configuration is A1. However, all of the models tend to show an increased noise level when compared to the A0 reference configuration of Figure 6.

Figure 9-Figure 10 isolate the effects of increasing the edge radius on the front and rear wheels individually. With only the front wheel rounded (Figure 9) there is significant increase in noise at all frequencies and for all model configurations. As for Figure 8, the noise increase is greatest for configuration B0 but there is also a 2-5dB noise increase for all configurations over most of the frequency spectrum. This increase in noise level peaks at around $4 \mathrm{kHz}$ but is also clearly observed below $2 \mathrm{kHz}$ and above $10 \mathrm{kHz}$. By contrast, rounding only the rear wheels produces a noise reduction between $2 \mathrm{kHz}$ and $6 \mathrm{kHz}$ and only small changes elsewhere in the spectrum (Figure 10).

Results from Figure 6-Figure 10 suggest that the effect of increasing front wheel radius from $10 \mathrm{~mm}$ to $20 \mathrm{~mm}$ is to raise the noise level across the frequency spectrum. This increase is typically between 2-4dB below frequencies of $8 \mathrm{kHz}$ and is exaggerated by increased separation between adjacent wheel pairs. However, increasing the rear wheel edge radius or increasing the lateral spacing between wheel pairs tends to offset this increase between 2-6kHz, with little effect on frequencies outside this range. Figure 7, Figure 11 also suggest that the peak source is located further towards the rear of the landing gear at lower frequencies. All the main sources are shown to be on the centerline of the landing gear although the array may have difficulty resolving two individual sources (on either side) in this region.

In Figure 12-Figure 16 a narrower $5 \mathrm{~mm}$ edge radius is applied to the front wheel. When the rear wheel edge radius remained at $10 \mathrm{~mm}$, the only significant noise increase was at the highest frequencies, where a source appeared towards the rear of the oleo / beam junction (Figure 13). However, when the rear wheel edge radius was changed to 20mm, a significant drop in overall noise level was observed, with the greatest noise reduction occurring between $2-6 \mathrm{kHz}$ (Figure 14). Source maps for these frequencies (Figure 15) show that the peak source at $2 \mathrm{kHz}$, which was close to the rear axle in Figure 7, Figure 11 has disappeared so that a secondary source close to the main strut has become dominant. The $5 \mathrm{~mm}$ front wheel edge radius and $20 \mathrm{~mm}$ rear wheel edge radius gave the lowest noise signature of all the models, interestingly with configuration B0 giving the lowest noise configuration.

Figure 16 demonstrates the effect of reversing the wheel pairs, with $20 \mathrm{~mm}$ front and $5 \mathrm{~mm}$ rear wheel edge radii, showing at least a 10dB increase in peak from the measurement of Figure 14.

\section{Isolation of noise interaction mechanisms}

The track configurations T0, T1 are comparable to models A0 and A1 of Figure 2. Comparison with the baseline configuration examined in Figure 6 shows a marked decrease in noise level at the centre of the frequency spectrum which is centered around $4000 \mathrm{~Hz}$, where a noise reduction of $8 \mathrm{~dB}$ is observed for model T0. Configuration T1shows a noise level which is around $2 \mathrm{~dB}$ higher than $\mathrm{T} 0$ across the spectrum, suggesting increased flow instability around the axles with the tracks further apart.

Model TOF1 represents the flow over a highly streamlined gear. The addition of an axle fairing to model T0 demonstrates a noise reduction of at least $4 \mathrm{~dB}$ beyond model $\mathrm{T} 0$ and across most of the frequency spectrum. Of particular note is the effect on sources below $2 \mathrm{kHz}$, whose levels are not reduced by the configurations of Figure 2 or by models $\mathrm{T} 0$ / $\mathrm{T} 1$.

\section{Twin wheel landing gears}

Figure 19 compares the noise level from a twin wheel landing gear with $10 \mathrm{~mm}$ wheel edge radius with the four wheel configuration of Figure 6. The two configurations (C0, C1) tend to show similar noise levels across the spectrum. Above $8 \mathrm{kHz}$, the noise produced by twin and four wheel gears is broadly the same as the four wheel gears. At these frequencies, most of the noise is produced by the fine joints and corners on the axles, which are similar on the twin and four wheel landing gears.

Comparison with models fitted with tracks (Figure 17) suggests a similar noise level between the twin wheel gears and model T0/T1 between $2-8 \mathrm{kHz}$ and highlights the noise benefit which may be available from eliminating the rear wheels and axles from the flow. At all frequencies the twin wheel gear is louder than configuration T0F1. 


\section{E. Summary of findings at model scale}

Results suggest that small changes to landing gear model geometry can achieve differences in noise level on the order of 10dB. In these experiments, shape of the wheel edges dominates the noise produced by the four wheel landing gear. Given a particular wheel shape, the effect of wheel layout may also have an important effect on the noise produced. Where the $20 \mathrm{~mm}$ wheel edge radius was used on the front wheels (Figure 8, Figure 9) and the streamwise spacing increased (configuration B) the noise level was found to increase by between 2-4dB in each third octave band. This is consistent with a complex wake developing between front and rear wheels, which is able to entrain high momentum flow from the free stream when the separation between adjacent wheels is larger. This hypothesis is supported by flow visualization measurements although these are beyond the scope of this paper.

For the four wheel landing gear, sources can be broadly separated into components below $2 \mathrm{kHz}$, between 2$6 \mathrm{kHz}$ and above $6 \mathrm{kHz}$.

\section{1. $1,000-2,000 H z$}

Below $2 \mathrm{kHz}$, the dominant source appears to be associated with the rear axle (Figure 7, Figure 11, Figure 13). This source is affected by the front wheel edge shape and the location of the rear axle. When the front wheel edge is changed from $10 \mathrm{~mm}$ to $20 \mathrm{~mm}$, this source is amplified by up to $4-6 \mathrm{~dB}$ (Figure 8, Figure 10). In Figure 12 there is a smaller noise increase when the front wheel edge is changed to $5 \mathrm{~mm}$. This was not repeatable in a similar experiment without the side strut, where measurements suggested very little change in noise at these frequencies.

Whenever the shorter beam is replaced with the longer beam (configuration A to B), there is an increase in low frequency noise on the order of $2 \mathrm{~dB}$. Changing the lateral separation of the wheels (configurations 0,1 ) or the shape of the rear wheel tends to have a relatively small effect on this source. Replacing the wheels with tracks (Figure 3 ) has little effect on this source until the axle fairing is added (model T0F1), when a noise reduction of at least 6dB is observed.

\section{2. $2 \mathrm{kHz}-6 \mathrm{kHz}$}

Noise in the $2-6 \mathrm{kHz}$ range of the model spectrum appears to be dominated by the shape of the rear wheels. Source maps indicate this source is associated with the inside edge of the rear wheels or the impingement of flow from the rear wheels on the main axle beam (Figure 7,Figure 11, Figure 13). Previous investigation of different wheel edge geometries with a fully faired landing gear (without axles or struts) would suggest the wheel edge is actually responsible in this case ${ }^{1}$.

The effect of rear wheel edge shape on the $2-6 \mathrm{kHz}$ source can be seen clearly in Figure 10, Figure 14. In Figure 10 , rounding the edge of the rear wheels from $10 \mathrm{~mm}$ to $20 \mathrm{~mm}$ gives a clear reduction in noise of between $2-4 \mathrm{~dB}$ in this frequency range. Previous experiments have demonstrated a similar reduction when changing from a $5 \mathrm{~mm}$ to a $10 \mathrm{~mm}$ wheel edge radius for a range of geometries ${ }^{1}$. A similarly well defined reduction can also be observed for the $5 \mathrm{~mm}, 20 \mathrm{~mm}$ case of Figure 14.

Although the source in the $2-6 \mathrm{kHz}$ range appears dominated by the shape of the rear wheels, it is also increased when a more rounded front wheel is used or when the distance between front and rear wheels is larger (configuration B). This source is also reduced when the lateral separation between wheels is increased (configuration 1), indicating more stability in the flow around the rear wheels. These effects are observed in Figure 8, Figure 9. In each case, the increase would be in agreement with a more energetic wake behind the front pair of wheels. This is expected to interact with the downstream wheel edge, where acoustic scattering is more significant for smaller curvatures. All configurations involving the track configuration (Figure 3) give a peak in noise reduction at $4-5 \mathrm{kHz}$ which directly corresponds to the wheel edge source.

\section{Above $6 \mathrm{kHz}$}

At the highest frequencies, the noise sources are generally found to the rear of the oleo / beam interface, as shown by source maps in Figure 7, Figure 11, Figure 13 and Figure 15. Above frequencies of $12.5 \mathrm{kHz}$, peak sources are also found in this region of the model. All of the models are designed without surface features on the axles or 
struts, so that the noise in this region of the spectrum is probably due to the junction of the main oleo / axle beam, which are at $90^{\circ}$ to each other, where the flow turns sharply into the sharp corner between oleo and main beam. In a fully dressed landing gear, this location would correspond directly to the location of the torque link, which may account for the high level of noise typically observed from this component.

Although the source of high frequency noise cannot be precisely determined, its amplitude is broadly coupled to the noise radiated at low frequencies for the four wheel models. Models showing a noise increase below $2 \mathrm{kHz}$ tend to also show a substantial increase in high frequency noise sources. In particular, it seems that rounding off the front wheels leads to higher local flow velocities and more unstable flow around the main strut, beam and axles. This increases noise at the lowest and highest frequencies.

\section{Twin wheel}

Results for the twin wheel landing gear appear to support this breakdown of source mechanisms. Elimination of the rear axle might provide the large reduction in noise below $2 \mathrm{kHz}$, whereas elimination of the rear pair of wheels removes the rear wheel edge geometry which has a significant effect between $2-6 \mathrm{kHz}$. Above frequencies of around $10 \mathrm{kHz}$, the twin and four wheel gears have a similar level of surface detail, which may explain the lack of noise reduction.

\section{F. Experimental Spectra}

Measurements from the microphone arrays can be used to form experimental spectra. However, attention must be paid to the varied sensitivity of the arrays at different frequencies, the directional dependence of the radiated sound, effects attributable to background noise and appropriate sampling and processing methods. The method used here is the same as discussed previously ${ }^{1}$.

The spectrum from the larger, low frequency array is generally at a lower level than that from the high frequency array. This difference can be attributed to the directivity of aerodynamic dipole sources and the shielding of sources from outer microphones of the low frequency array by the presence of wheels.

However, one of the most useful observation points is directly below the landing gear as this is the closest point for any ground observer so that the small footprint of the high frequency array makes its level more suitable. To form a complete spectrum, the low frequency spectra are shifted to intersect with high frequency data in the $2-4 \mathrm{kHz}$ range. The spectrum is then read from the adjusted low frequency array data below 6kHz, and from the high frequency array data above $6 \mathrm{kHz}$, as shown in Figure 20. Also shown in Figure 20 are error bars corresponding to a single standard deviation, as measured from three individual measurements.

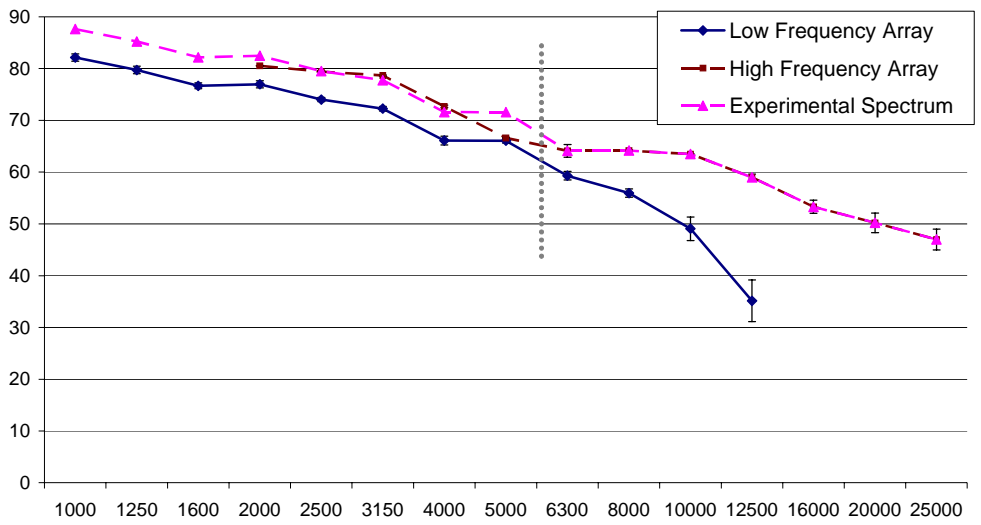

Figure 20 - Experimental spectra from both acoustic arrays (Model A0) 


\section{G. Application to full scale landing gears}

Once model scale spectra have been defined, these can be approximated for a full size landing gear with similar geometry. Whilst results measured from these models may not scale exactly to full size, it is valuable to look at how the findings here might influence the noise at full scale. Noise measurements from a typical aerodynamic landing gear source can be expected to scale in a dipole manner ${ }^{14}$ :

$$
\begin{gathered}
S P L=S P L_{\text {model }}+20 \log 10\left(d_{\text {aircraft }} / d_{\text {model }}\right)+60 \log 10\left(M_{\text {aircraft }} / M_{\text {model }}\right)-20 \log 10\left(r_{\text {aircraft }} / r_{\text {model }}\right) \\
f=f_{\text {model }} \times \frac{V_{\text {aircraft }} d_{\text {model }}}{V_{\text {model }} d_{\text {aircraft }}}
\end{gathered}
$$

Results are scaled to a flow speed of $60 \mathrm{~m} / \mathrm{s}$ and aircraft wheel diameter of $1.2 \mathrm{~m}$, providing a factor of 10 between model scale frequencies and those anticipated at full scale. Figure 21-Figure 23 demonstrate the effects of the wheel shape and location on the noise predicted at full scale for A-weighted spectra. Wheel edge radii shown in these spectra refer to model scale values and all spectra include the four wheel A0 configuration with a 10mm wheel edge as a reference line (in red).

Figure 21 also shows the predicted noise from a single main landing gear using the component based prediction tool by Guo ${ }^{8}$. This method uses details of the gear geometry to determine an estimate of overall noise level. The estimates in Figure 21 are for a fully dressed conventional gear and for a simplified landing gear similar to those of Figure 2.

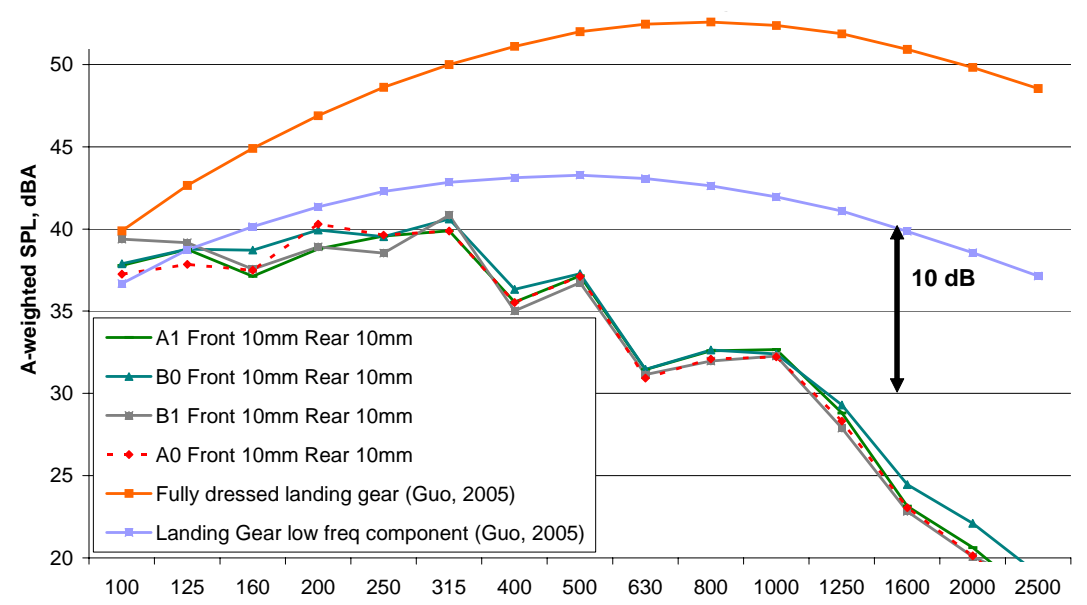

Figure 21 - A-weighted spectra for four wheel gears with edge rounding 10mm (Figure 6) (Wheel edge radii refer to model scale values)
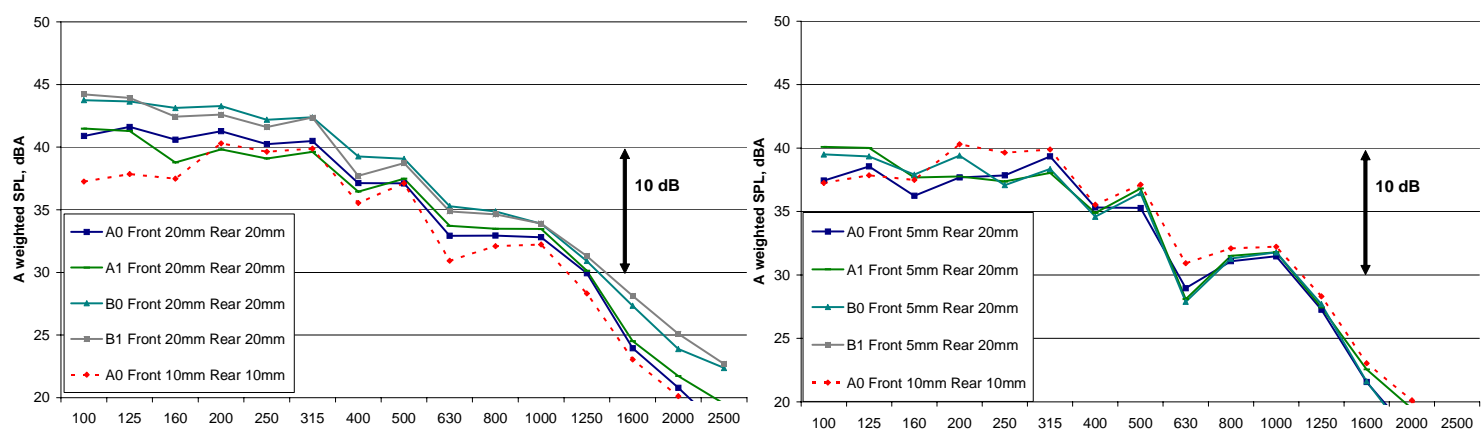

Figure 22 - A-weighted spectra for four wheel gears with different wheel edge shapes (Figure 8 / Figure 14)

(Wheel edge radii refer to model scale values) 
The noise level predicted from the A0 landing gear of Figure 21 is approximately $14 \mathrm{dBA}$ below that anticipated from fully dressed conventional gears under the same conditions. Most of this noise reduction comes from elimination of high frequency details and surface discontinuities, which contribute significantly to the spectrum above $500 \mathrm{~Hz}$. At the lowest frequencies, scaled wind tunnel data tend to agree with predictions from the noise model for a simplified gear. However, above $400 \mathrm{~Hz}$ the spectra depart from the predictive model. This can be attributed to the highly simplified external geometry of the current models (Figure 2) when compared to those used to fit prediction model parameters ${ }^{8}$.

For the different configurations of Figure 2, the largest reduction in four wheel gear noise comes from changing the shape of the landing gear wheels (Figure 21, Figure 22). Increased rounding of all wheels on the landing gear increases overall noise by 2-4dBA, depending on wheel spacing (Figure 22a), with the most striking increase at the lowest measured frequencies (corresponding to $100 \mathrm{~Hz}$ full scale). Increasing the lateral spacing also tends to reduce noise between 125-400Hz in Figure 21, Figure 22a although these effects are weak in comparison to the wheel edge effect.

Figure 22b shows the effect of designing wheel shape for the lowest noise configuration. When compared to the baseline configuration, a noise reduction of approximately $2 \mathrm{dBA}$ can be achieved close to the peak of the spectrum by shaping the wheels to appropriately control the flow.

The effect of gaps between adjacent wheels is studied in Figure 23a. Configurations T0 / T1 demonstrate up to $5 \mathrm{dBA}$ noise reduction in the centre of the spectrum $(200-1000 \mathrm{~Hz})$ but are unable to reduce noise at the lowest frequencies. By isolating the axles in configuration T0F1 (Figure 3), a drop in overall noise of at least 6dBA is observed. In particular, noise below $300 \mathrm{~Hz}$ is reduced by up to $8 \mathrm{dBA}$.
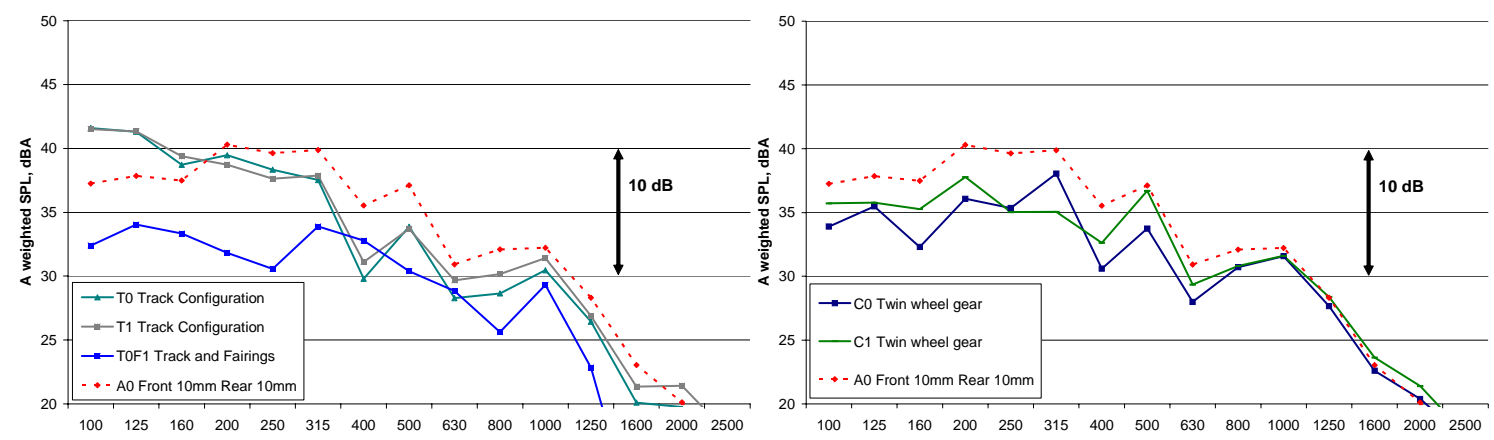

Figure 23 - A-weighted spectra for the (a) track and (b) twin wheel configurations (Figure 17 / Figure 19)

Twin wheel configurations (Figure 23b) demonstrate 3-5dBA reduction in noise below $800 \mathrm{~Hz}$, with the largest noise reduction at the peak of the four wheel spectrum, resulting in at least 3dBA reduction in overall A-weighted SPL. The noise levels observed are not as low as from model T0F1, reflecting the confined axle geometry of the twin wheel gear (Figure 5).

\section{Extension to include smaller details.}

Although the above figures show wind tunnel measurements scaled to full size and speed, they do not account for the increased complexity which would be present on a full size landing gear (Figure 21). Analysis of the different components of sound generated by these models (Section IV.E) suggests that flow instabilities at both low and high frequencies are coupled to the basic design of the landing gear. The ability to reduce low frequency noise by making small changes to the gear geometry as outlined in Figure 2 may allow a significant proportion of the high frequency noise associated with dressings to be reduced without compromising the design integrity of the landing gear. These effects cannot be predicted from the information in these experiments but may lead to a far more significant landing gear noise reduction than shown by Figure 21-Figure 23. 


\section{Conclusions}

Simplified twin and four wheel landing gear models have been examined successfully using a pair of acoustic arrays. Design of the models eliminated all geometric details and provided a smooth external surface, allowing isolation of sources which are more fundamental to the basic design of the landing gear. Models were also constructed to allow variations in the basic geometry, including the ability to vary wheel shape and location. Further simplifications allowed isolation of the interaction between adjacent wheels and the noise sources associated with axles.

Experiments with the four wheel landing gear model (Figure 2) show that the location and shape of the wheels can have a significant effect on overall noise levels. Increasing the edge radius of the front wheel pair tends to increase the noise level seen from the landing gear across the whole frequency spectrum, in some cases by as much as $6 \mathrm{~dB}$. The layout of the wheels also determines the magnitude of noise increase. In the baseline configuration, the separation between wheels shows only a small influence on landing gear noise. However, when the front wheel edge radius is increased, a larger separation between front and rear wheels leads to a much more severe noise increase. This is consistent with the development of a more powerful wake between the wheels which can entrain external high speed flow. Increasing the wheel edge radius on the rear wheels or the lateral separation between the wheels also tends to decrease noise levels.

Isolation of noise sources through application of 'tracks' and axle fairings as well as analysis of the twin wheel landing gear suggests that different sources dominate different areas of the noise spectrum. At model scale, noise generated below $2 \mathrm{kHz}$ ( $200 \mathrm{~Hz}$ full scale) is shown to be generated near to the rear axle. This source is increased when a softer wheel edge radius is used on the front wheel, indicating an interaction between the front wheel wake and the rear axle. Noise at these frequencies is unaffected or increased by using a 'track' configuration but reduced by using an axle fairing or twin wheel configuration.

Noise at $2-6 \mathrm{kHz}(200-600 \mathrm{~Hz}$ full scale) is particularly affected by the rear wheel edge geometry on the four wheel model. Source maps indicate the main source at these frequencies to be located near the front inside edge of the rear wheels. Effects are generally between $2-4 \mathrm{~dB}$ for the geometries chosen here. The installation of 'tracks' instead of wheels (Figure 3) is shown to be a particularly effective for noise reduction at these frequencies. This source is not present on twin wheel landing gears, which are typically $6 \mathrm{~dB}$ quieter than the four wheel gears at these frequencies for the configurations examined here.

At frequencies above $6 \mathrm{kHz}$, sources were located close to the oleo / beam junction of the four wheel gear. The amplitude of noise at these frequencies is also dependent on the shape of the front wheels, with much stronger sources observed when the edge of the front wheels is more rounded. This suggests that flow stability around the oleo / beam junction is at least partially determined by the shape of the wake from the front wheel. On a fully dressed gear, the area close to the oleo / beam junction is an important structural one, and generally contains a torque link and a large number of brake components and fixings. The ability to control the flow around this area of the gear by appropriate shaping and placing of the wheels may prove useful in the mitigation of higher frequency noise on more conventional landing gears.

\section{Acknowledgments}

The authors would like to express their thanks to Mr John Clark for manufacture of all models examined in the course of this work. The authors would also like to thank Dr. Pieter Sijtsma of NLR for his continued support and interest in results obtained from the acoustic array in Cambridge. 


\section{References}

${ }^{1 .}$ Quayle, A. R., Dowling, A. P., Babinsky, H., Graham, W., and Sijtsma, P., Landing Gear for a Silent Aircraft, in 45th AIAA Aerospace Sciences Meeting and Exhibit AIAA Paper 2007-0231, Reno, NV, 2007.

2. Dobrzynski, W. M., Chow, L. C., Guion, P., and Shiells, D., A European Study on Landing Gear Airframe Noise Sources, in 6th AIAA/CEAS Aeroacoustics Conference AIAA 2000-1971, 2000.

3. Stoker, R. W. and Sen, R., An Experimental Investigation of Airframe Noise Using a Model Scale Boeing 777, in 39th AIAA Aerospace Sciences Meeting \& Exhibit AIAA Paper 2001-0987, Reno, NV, 2001.

4. Jaeger, S. M., Burnside, N. J., Soderman, P. T., Clifton Horne, W., and James, K. D., Microphone Array Assessment of an Isolated 26\%-scale, High Fidelity Landing Gear, in 8th AIAA/CEAS Aeroacoustics Conference and Exhibit AIAA 2002-2410, Breckenridge, Colorado, 2002.

${ }^{5 .}$ Ravetta, P., Burdisso, R., and Ng, W., Wind Tunnel Measurements of a 26\%-scale 777 Main Landing Gear Model, in 10th AIAA/CEAS Aeroacoustics Conference AIAA Paper 2004-2885, 2004.

6. Smith, M. G., Fenech, B., Choi Chow, L., Molin, N., Dobrzynski, W., and Seror, C., Control of Noise Sources on Aircraft Landing Gear Bogies, in 12th AIAA/CEAS Aeroacoustics Conference (27th AIAA Aeroacoustics Conference) AIAA Paper 2006-2626, Cambridge, MA, 2006.

7. ESDU, Engineering Science Data Unit (UK) - Airframe Noise Prediction. Item No 90023., 1992.

${ }^{8 .}$ Guo YP, Empirical Prediction of Aircraft Landing Gear Noise, NASA CR-2005-213780, 2005.

${ }^{9}$ Lazos, B. S., "Mean Flow Features Around the Inline Wheels of Four-Wheel Landing Gear," AIAA Journal, Vol. 40, No. 2, 2002, pp. 193-198.

10. Shin, H., Graham, W., and Faszer, A., Design and Implementation of a Phased Microphone Array in a Closed-Section Wind Tunnel in 12th AIAA/CEAS Aeroacoustics Conference (27th AIAA Aeroacoustics Conference) AIAA-2006-2651, Cambridge, Massachusetts, 2006.

11. Sijtsma, P., Experimental Techniques for Identification and Characterisation of Noise Sources, in Advances in Aeroacoustics and Applications, VKI Lecture Series, 2004-05.

12. Quayle, A. R., Dowling, A. P., Graham, W. R., and Babinsky, H., Comparison of Source Estimation Algorithms / Methods in Closed Tunnel Noise Measurements, in Berlin Beamforming Conference (BeBeC) 2006 TU Berlin, Berlin, Germany, 2006.

13. Högbom, J. A., "Aperture Synthesis with a Non-Regular Distribution of Interferometer Baselines," Astron. Astrophys. Suppl., Vol. 15, No., 1974, pp. 417-426.

14. Dowling, A. P. and Ffowcs-Williams, J. E., Sound and Sources of Sound Ellis Horwood Publishers, 2000. 